\title{
Are There Silicate-S Stars?
}

\author{
IRENE R. LITTLE-MARENIN \\ Wellesley College, Wellesley MA, U.S.A.
}

I have identified seven $\mathrm{S}$ stars with very strong silicate features in their IRAS LRS spectra at 10 and $18 \mu \mathrm{m}$ (LRS 25-29) (Chen et al. 1995, $A \forall A S$, $113,51)$. This is highly unusual since $\mathrm{S}$ stars tend to have lower mass-loss rates and higher gas-to-dust ratios than $\mathrm{M}$ or $\mathrm{C}$ stars, implying less efficient dust formation in their circumstellar shells. Gas-to-dust ratios are estimated to be between 400 and 1000, at least a factor of two lower than for carbon stars, and hence strong dust emission features are not seen or expected. Also, pure S stars have a relatively weak emission feature which peaks in the 10-11 $\mu \mathrm{m}$ region and is subtly different from the $10 \mu \mathrm{m}$ silicate or the $11.2 \mu \mathrm{m} \mathrm{SiC}$ feature. However, all seven stars have been found to be either M or MS stars rather than pure $S$ stars, and hence they reflect the mass-loss rates and dust content associated with $\mathrm{M}$ stars. The stars and their characteristics are listed below.

Table 1: S? Stars with Strong Silicate Emission Features

\begin{tabular}{ccrcrcl}
\hline IRAS & Name & GCSS & LRS & \multicolumn{1}{c}{$l$} & \multicolumn{1}{c}{$b$} & Sp. Class \\
\hline $07197-1451$ & TT CMa & 341 & 27 & 230 & -0.2 & not S, maybe MS (a) \\
$11169-6111$ & & 738 & 29 & 292 & -0.5 & M5.5, not S (b) \\
$15347-5555$ & & 897 & 26 & $325-0.5$ & M3 (b) \\
$16490-4618$ & & 944 & 25 & $340-1.5$ & M1.5 (b) \\
$19545-1122$ & V1407 Aql & 1175 & 29 & $30-19.6$ & M6S (c) \\
$21029+4917$ & & 1259 & 28 & $90+1.7$ & M3 (d) \\
$22512+6100$ & V386 Cep & 1314 & 28 & $109+1.6$ & M6 (c), M3 (a) \\
\hline
\end{tabular}

(a) Stephenson, private communication; (b) Lloyd Evans \& Catchpole 1989, MNRAS, 237, 219; (c) Bidelman, private communication; (d) Cohen et al. 1989, AJ, 97, 1759 